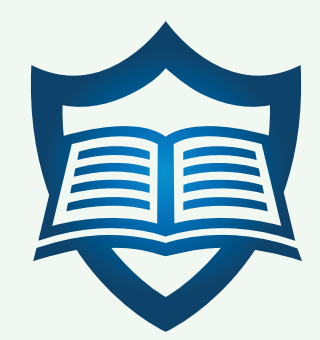

\title{
American Journal of
}

\section{Epidemiology \& Public Health}

\section{Short Communication}

\section{Forecasting COVID-19 Cases and Deaths in Epidemic-Mitigating European Countries} by Richards Function-Based Regression Analyses - $\odot$

\section{Zhifu FU', Xinmiao $\mathrm{FU}^{2 *}$ and Cheng LONG ${ }^{3 *}$}

'Anxi AIER Eye Hospital, Anxi County, Fujian Province 362400, China

${ }^{2}$ College of Life Sciences, Fujian Normal University, Fuzhou City, Fujian Province 350117, China

${ }^{3}$ Department of Orthopaedic, Sichuan University West China Hospital, Chengdu City, Sichuan Province 610041 , China

*Address for Correspondence: Xinmiao Fu, College of Life Sciences, Fujian Normal University, Fuzhou City, Fujian Province 3501 17, China, ORCID ID: orcid.org/0000-0003-3361-6904; E-mail: xmfu@fjnu.edu.cn

Cheng Long, Department of Orthopaedic, Sichuan University West China Hospital, Chengdu City, Sichuan Province 610041, China, E-mail: longchenghx@126.com

Submitted: 12 June 2020; Approved: 17 June 2020; Published: 17 June 2020

Cite this article: FU Z, FU X, LONG C. Forecasting COVID-19 Cases and Deaths in Epidemic-Mitigating European Countries by Richards Function-Based Regression Analyses. American J Epidemiol Public Health. 2020;4(2): 048-051. https://dx.doi.org/10.37871/ajeph.id28

Copyright: @ $2020 \mathrm{FU}$ Z, et al. This is an open access article distributed under the Creative Commons Attribution License, which permits unrestricted use, distribution, and reproduction in any medium, provided the original work is properly cited. 


\begin{abstract}
The COVID-19 pandemic has hit many countries, and people raise grave concerns about the trajectory of COVID-19 outbreaks that profoundly changed the societal and economic activities in these countries. Richards function, which is suitable for predicting the final outbreak size shortly after the peak time, was applied here to simulate and forecast the course of COVID-19 outbreaks in 10 epidemicmitigating European countries, i.e., Italy, Spain, France, Germany, Turkey, Belgium, Ireland, Netherlands, Portugal and Switzerland, based on their daily cumulative confirmed cases as of May 15, 2020. Potential total COVID-19 confirmed cases in these countries were estimated to be $240400 \pm 1300,294100 \pm 4000,178500 \pm 800,176900 \pm 700,155400 \pm 1000,57900 \pm 400,24000 \pm 200,46200 \pm 300,30000 \pm 300$ and $30700 \pm 100$, respectively. Most of these countries are predicted to approach ending stage between late May and early June such that daily new cases will become minimal. Total COVID-19 deaths were also estimated by Richard function-based analysis. Our estimated total cases and deaths agree well with real data in these countries as of June 15, 2020. To our best knowledge, this is the first study forecasting the COVID-19 epidemic by applying the Richard function-based regression analysis.
\end{abstract}

\section{INTRODUCTION}

The COVID-19 pandemic caused by the novel coronavirus (SARS-CoV-2) has hit many countries [1], resulting in more than 4 million cases and over 300,000 deaths in the world as of May 14, 2020. In European countries such as Italy and Spain, daily new cases are declining since April, yet they are still as many as one thousand per day. In these epidemic-mitigating countries, governments are attempting to restore normal societal and economic activities and concerns are raised about the risk of COVID-19 resurgence. It is of significance to precisely forecast when the epidemic in each country will become minimal such that societal and economic restorations will not lead in the resurgence of the outbreak. Further, such forecasting may also inform governments to get better preparedness for implementing control measures and supplying medical resources.

Epidemic forecasting by traditional epidemiological models apparently require much detailed data for analysis [2,3]. Here we applied a simple data-driven, Richards function-based approach for epidemic forecasting only based on the daily cumulative confirmed COVID-19 cases. The Richards function is intrinsically linked to the traditional epidemic Susceptible-Infectious-Recovered (SIR) model and can predict the final outbreak size shortly after the peak time [4]. As such, this model is suitable for simulating and forecasting the course of COVID-19 in epidemic-mitigating countries, in which the peak of daily new cases has passed. In retrospect, Richards functionbased analysis has been explored for simulating 2003 SARS outbreaks in several regions/countries by others $[4,5]$ and also for estimating COVID-19 deaths in China by us recently [6].

\section{METHODS}

According to earlier reports [4,5], the Richards function is expressed as follows:

$$
y=a\left[1+(d-1) e^{k(t-t c)}\right]^{\left(\frac{1}{1-d}\right)}
$$

where $y$ is the cumulative number of confirmed COVID-19 cases and $t$ is time (days); $a, d$, tc and $k$ are constants. Simulation was performed using Microcal Origin software with the Richard function. Initial values of $d, t c$ and $k$ for the simulation were set as 4,60 and 0.5 , respectively, based on our experience, and $a$ was set as the number of cumulative confirmed cases of each country on May 14, 2020. In particular, $t c$ represents the turning point [4], on which daily new cases are declining. It should be pointed out that the simulation process is robust such that best parameters can be obtained after several rounds of calculations. Total number of COVID-19 cases is given by parameter $a$ in the equation.

\section{RESULTS}

\section{Estimation of potential total COVID-19 confirmed cases in each country}

We decided to collect the data of the cumulative confirmed cases from the website of Worldometer [7] for 10 most affected, epidemicmitigating European countries, i.e., Italy, Spain, France, Germany, Turkey, Belgium, Ireland, Netherlands, Portugal and Switzerland (for detail, refer to the supporting information file original data. xlsx), in which daily new cases are significantly declining since late April and/or early May. In comparison, UK and Sweden, two most affected European countries with more than 240,000 and 29,000 COVID-19 confirmed cases as of May 15, respectively, were not subjected to analysis because their daily new cases have not yet started to significantly decline (Figure S1) and do not meet the requirement for Richard function-based analysis (i.e., the peak time of the subject has passed [4]).

Regression analyses indicate that the data of each country were well fitted with the Richards function (all $R^{2}$ values being close to 0.999; table 1 and figures $1 \mathrm{~A}$ and $1 \mathrm{~B}$ ). Potential total numbers of confirmed cases in the above countries were estimated to be 240400 $\pm 1300,294100 \pm 4000,178500 \pm 800,176900 \pm 700,155400 \pm 1000$, $57900 \pm 400,24000 \pm 200,46200 \pm 300,30000 \pm 300$ and $30700 \pm 100$, respectively. All parameters from regression analyses are presented in table $\mathrm{S} 1$ and can be utilized to predict daily new cases in each country since May 15 according to equation 1.

For comparison, we subjected the same data to Boltzmann function-based regression analysis, which has been explored by us to precisely estimate the COVID-19 outbreaks in China (including Hubei Province and Wuhan City, epicenter of COVID-19 outbreaks in China) based on the data as of February 14, 2020 [8]. Nevertheless, Boltzmann function-based analyses appear to underestimate the potential total numbers of COVID-19 cases in the above countries (refer to black curves in figure 1A). These results suggest that the Richards function is more suitable than the Boltzmann function to simulate and forecast the COVID-19 epidemics in these countries. In addition, we estimated key date, on which daily new cases will be lower than $0.1 \%$ of potential total confirmed cases as defined by us subjectively. It appears that most countries will approach such ending stage between late May and early June (refer to table 1) such that daily new cases will become minimal in comparison with potential total confirmed cases. 

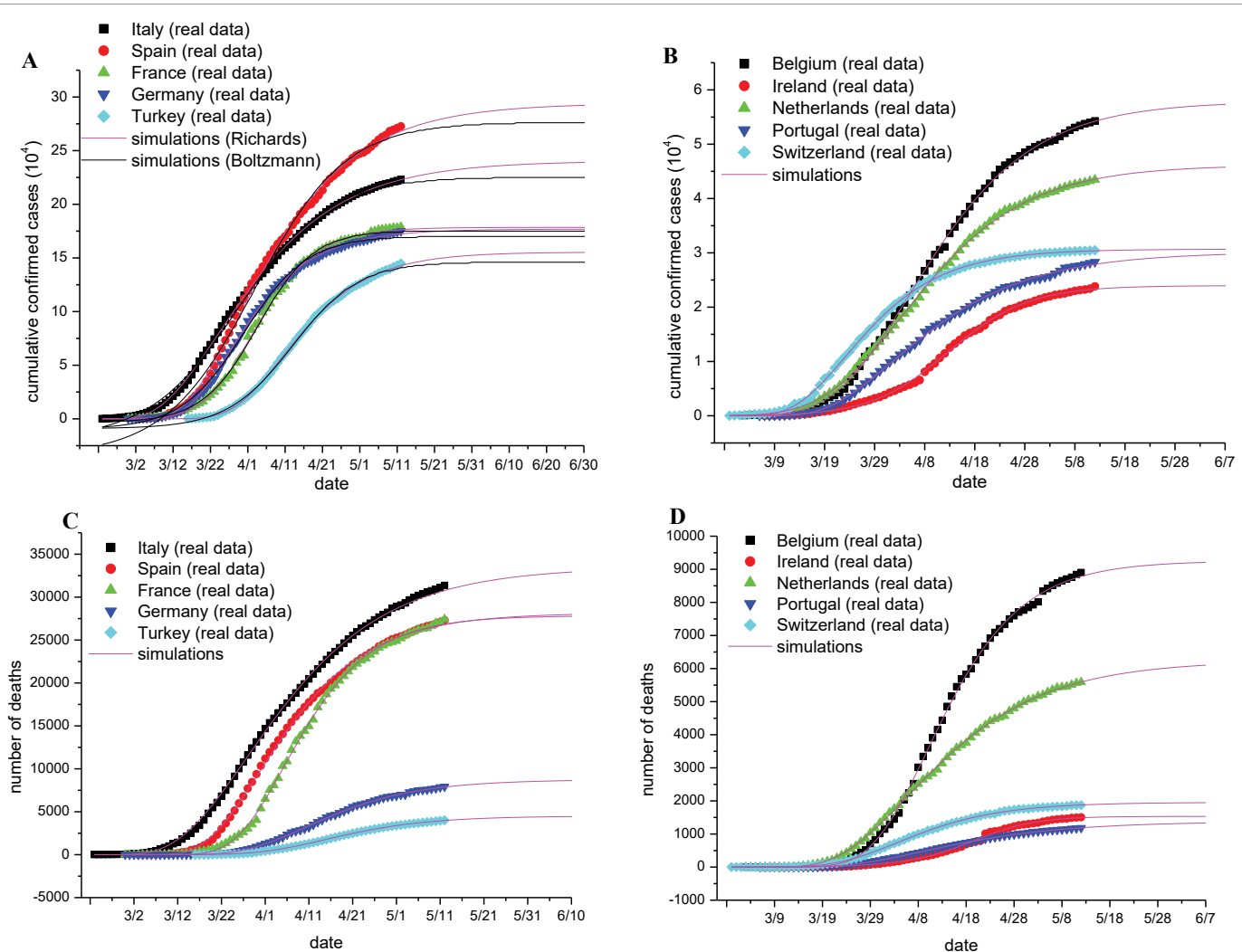

Figure 1: Fitting and forecasting the cumulative confirmed COVID-19 cases and deaths of each European country with the Richards function.

(A, B) Plots of the cumulative number of COVID-19 confirmed cases of the indicated European countries as of May 14, 2020, with the simulation results being plotted as Magenta lines. Red and black curves in panel A represent the Richards function- and Boltzmann function-based regression analysis results. (C, D) Plots of the cumulative number of COVID-19 confirmed cases of the indicated European countries as of May 14, 2020, with the simulation results being plotted as Magenta lines. Simulation was performed using Microcal Origin software with the Richard function. All parameters from regression analyses are shown in table $\mathrm{S} 1$ (for cases) and table S2 (for deaths).

Table 1: Estimation of potential total COVID-19 confirmed cases and deaths in European countries.

\begin{tabular}{|c|c|c|c|c|c|c|c|c|}
\hline \multirow{2}{*}{ Countries } & \multicolumn{2}{|c|}{ Total confirmed cases } & \multirow{2}{*}{ Key date ${ }^{a}$} & \multicolumn{2}{|c|}{ Total deaths } & \multirow{2}{*}{$\begin{array}{l}\text { Crude fatality } \\
\text { ratio }(\%)^{b}\end{array}$} & \multicolumn{2}{|c|}{ Real data (as of June 15) } \\
\hline & Number & $R^{2}$ & & Number & $R^{2}$ & & $\begin{array}{l}\text { Total confirmed } \\
\text { cases }\end{array}$ & Total deaths \\
\hline Italy & $\begin{array}{l}240,400 \pm \\
1,300\end{array}$ & 0.999 & June 6 & $33,500 \pm 300$ & 0.999 & $13.9 \pm 0.9$ & 236,989 & 34,345 \\
\hline Spain & $\begin{array}{l}294,100 \pm \\
4,000\end{array}$ & 0.999 & June 13 & $28,200 \pm 200$ & 0.999 & $9.6 \pm 0.9$ & 291,008 & 27,136 \\
\hline France & $178,500 \pm 800$ & 0.999 & May 18 & $27,800 \pm 200$ & 0.999 & $15.6 \pm 0.7$ & 157,220 & 29,407 \\
\hline Germany & $176,900 \pm 700$ & 0.999 & May 24 & $8,740 \pm 80$ & 0.999 & $4.9 \pm 0.9$ & 186,461 & 8,791 \\
\hline Turkey & $155,400 \pm 1000$ & 0.999 & June 8 & $4,500 \pm 30$ & 0.999 & $2.9 \pm 0.7$ & 178,239 & 4,807 \\
\hline Belgium & $57,900 \pm 400$ & 0.999 & June 5 & $9,250 \pm 70$ & 0.999 & $16.0 \pm 0.9$ & 60,029 & 9,655 \\
\hline Ireland, & $24,000 \pm 200$ & 0.998 & May 25 & $1,530 \pm 20$ & 0.997 & $6.4 \pm 1.3$ & 25,303 & 1,706 \\
\hline Netherlands & $46,200 \pm 300$ & 0.999 & June 4 & $6,240 \pm 50$ & 0.999 & $13.5 \pm 0.9$ & 48,783 & 6,059 \\
\hline Portugal & $30,000 \pm 300$ & 0.999 & June 9 & $1,380 \pm 10$ & 0.999 & $4.6 \pm 0.8$ & 36,690 & 1,517 \\
\hline Switzerland & $30,700 \pm 100$ & 0.999 & May 14 & $1,960 \pm 8$ & 0.999 & $6.4 \pm 0.4$ & 31,034 & 1,676 \\
\hline
\end{tabular}

\section{Estimation of potential total COVID-19 deaths}

In several countries, crude fatality ratios are as high as over $10 \%$ such that people have raised grave concerns about how many patients will die eventually. With respect to each country under specific circumstances, the number of COVID-19 deaths can be assumed to be proportional to the number of confirmed cases. Along this logic thinking, we speculated that the cumulative COVID-19 deaths would also obey the Richards function. Regression analyses indicate that the data of cumulative COVID-19 deaths in each country were well fitted with the Richards function (all $R^{2}$ values being close to 0.999; table 
1 and figures 1C and 1D). Potential total COVID-19 deaths in Italy, Spain, France, Germany, Turkey, Belgium, Ireland, Netherlands, Portugal and Switzerland were estimated to be $33500 \pm 300,28200 \pm$ $200,27800 \pm 200,8740 \pm 80,4500 \pm 30,9250 \pm 70,1530 \pm 20,6240 \pm$ $50,1380 \pm 10$ and $1960 \pm 8$, respectively. Crude fatality ratios (total deaths among total confirmed cases) of these countries could be estimated to be $13.9 \pm 0.9 \%, 9.6 \pm 0.9 \%, 15.6 \pm 0.7 \%, 4.9 \pm 0.9 \% .2 .9$ $\pm 0.7 \%, 16.0 \pm 0.9 \%, 6.4 \pm 1.3 \%, 13.5 \pm 0.9 \%, 4.6 \pm 0.8 \%$ and $6.4 \pm$ $0.4 \%$, respectively. All parameters of regression analyses are displayed in table S2 and can be utilized to predict daily new deaths in each country since May 15 according to equation 1 .

\section{DISCUSSION}

To our best knowledge, this is the first study forecasting the COVID-19 epidemic by applying Richard function-based regression analysis. It should be pointed out that the Richard function-based estimates are based on the assumption that there are no significant changes in the current interventions, including non-pharmacological interventions [9] such as quarantine [10,11], other prevention measures [12], mass testing [13] and also diagnosis and treatment approaches $[14,15]$.

The estimated potential total COVID-19 confirmed cases and deaths are relatively accurate when compared with the real data of each country as of June 15, 2020 (Table 1), indicating that the Richards function is suitable for forecasting COVID-19 outbreak sizes in epidemic-mitigating countries. Therefore, it of interest to apply this approach for analyzing COVID-19 outbreaks in other most affected, epidemic-mitigating countries. Collectively, our forecasting on the course of COVID-19 outbreaks in these European countries may provide guidance for governments and also the public to get better preparedness and optimize their efforts to contain this unprecedented crisis at current critical stage. The estimated key date for each country may serve as a reference for operating societal and economic restorations such that the resurgence of COVID-19 can be avoided, minimized or under control according to COVID-19 containment in China $[16,17]$. In particular, the initiation of nationwide societal and economic restorations should be executed, at least, not earlier than the estimated key dates, although local restorations may be operated earlier according to the local epidemic situation.

Our prediction on the course of COVID-19 deaths (refer to figures 1C, 1D and table 1, S2) may benefit the mental health service that needs to be timely provided to the families of passed patients [18], given that thousands of COVID-19 patients have died in these countries and hundreds are dying at present. As a matter of fact, we precisely estimated total COVID-19 deaths in different areas of China (including Hubei Province and Wuhan city, the epicenter of the COVID-19 outbreaks in China [1]) by applying both Boltzmann and Richards functions-based regression analyses [6]. Such estimation helped Chinese governments to evaluate the severity of the COVID-19 epidemics.

\section{ACKNOWLEDGMENTS}

This work is support by the National Natural Science Foundation of China (No. 31972918 and 31770830 to XF). We thank graduate students (Zuqin Zhang, Youhui Zeng, Shuting Shi and Zhijie Huang, all from Professor Fu's research group at Fujian Normal University) for data collection.

\section{AUTHOR CONTRIBUTION}

X.F. conceptually designed the study; C.L. organized data and performed analysis; Z.F. wrote the manuscript; X.F. made the revision.

\section{Supplementary Files (Downloadable)}

\section{REFERENCES}

1. WHO: Coronavirus disease (COVID-19) outbreak. https://bit.ly/3hDEu0S.

2. Wu JT, Leung K, Leung GM. Nowcasting and forecasting the potential domestic and international spread of the 2019-nCoV outbreak originating in Wuhan, China: A modelling study. Lancet. 2020; 395: 689-697. DOI: https:// doi.org/10.1016/S0140-6736(20)30260-9

3. Yang Y, Lu Q, Liu M, Wang Y, Zhang A, Jalali N, et al. Epidemiological and clinical features of the 2019 novel coronavirus outbreak in China. 2020. DOI: https://doi.org/10.1101/2020.02.10.20021675

4. Wang XS, Wu J, Yang Y, et al. Richards model revisited: Validation by and application to infection dynamics. J Theor Biol. 2012; 313: 12-19. DOI: https:// doi.org/10.1016/j.jtbi.2012.07.024

5. Hsieh YH. Richards Model: A simple procedure for real-time prediction of outbreak severity. Modeling and Dynamics of Infectious Diseases. 2009; 216236. DOI: doi/abs/10.1142/9789814261265_0009

6. Gao Y, Zhang Z, Yao W, Ying Q, Long C, Fu X. (2020) Forecasting the cumulative number of COVID-19 deaths in China: A Boltzmann functionbased modeling study. Infect Control Hosp Epidemiol. 2020; 1-3. DOI: https:// doi.org/10.1017/ice.2020.101

7. Worldometer: COVID-19 Coronavirus pandemic. https://www.worldometers. info/coronavirus/

8. Fu X, Ying $Q$, Zeng $T$, Long $T$, Wang $Y$. Simulating and forecasting the cumulative confirmed cases of SARS-CoV-2 in China by Boltzmann functionbased regression analyses J Infect. 2020; 80: 578-606. PubMed: https:// pubmed.ncbi.nlm.nih.gov/32112887/

9. Pan A, Liu L, Wang C, Guo H, Hao X, Wang Q, et al. Association of public health interventions with the epidemiology of the COVID-19 outbreak in Wuhan, China. JAMA. 2020; 323: 1-9. https://doi.org/10.1001/jama.2020.6130

10. Alfano V, Ercolano S. The efficacy of lockdown against COVID-19: A crosscountry panel analysis. Applied Health Economics and Health Policy. 2020; DOI: https://dx.doi.org/10.1007\%2Fs40258-020-00596-3

11. Lau H, Khosrawipour V, Kocbach P, Mikolajczyk A, Schubert J, Bania J, et al. The positive impact of lockdown in Wuhan on containing the COVID-19 outbreak in China. J Travel Med. 2020; 27: 037. DOI: https://doi.org/10.1093/ jtm/taaa037

12. Hsiang S, Allen D, Annan-Phan S, Bell K, Bolliger I, Chong T, et al. The effect of large-scale anti-contagion policies on the COVID-19 pandemic. Nature. 2020; https://go.nature.com/3hxkyNk

13. Piguillem F, Shi L. The optimal covid-19 quarantine and testing policies (No. 2004). Einaudi Institute for Economics and Finance (EIEF). 2020.

14. Zhang, K., et al. Clinically applicable Al system for accurate diagnosis, quantitative measurements, and prognosis of COVID-19 pneumonia using computed tomography. Cell. 2020; 181: 1423-1433. DOI: https://doi. org/10.1016/j.cell.2020.04.045

15. Beigel JH, Tomashek KM, Dodd LE, Mehta AK, Zingman BS, Kalil AC, et al. Remdesivir for the treatment of Covid-19-preliminary report. $\mathrm{N}$ Engl J Med. 2020; DOI: https://doi.org/10.1056/NEJMoa2007764

16. Report of the WHO-China joint mission on coronavirus disease 2019 (COVID-19). 2020. https://bit.ly/3hFdajc

17. National Health Commission of China: Update of the COVID-19 outbreak data (in Chinese).

18. Xiang YT, et al. Timely mental health care for the 2019 novel coronavirus outbreak is urgently needed. Lancet Psychiatry. 2020; 7: 228-229. https:// bit.ly/3ddcwFU 